\title{
Les marins du Plessis-Bertrand à l'époque de Louis XIV : les opportunités d'une base de données
}

The sailors of Plessis-Bertrand under Louis XIV : the opportunities offered by a database

\section{Aurélie Hess et Philippe Hrodĕj}

\section{(2) OpenEdition}

\section{Journals}

Édition électronique

URL : https://journals.openedition.org/abpo/4358

DOI : $10.4000 / a b p o .4358$

ISBN : 978-2-7535-7783-1

ISSN : 2108-6443

\section{Éditeur}

Presses universitaires de Rennes

\section{Édition imprimée}

Date de publication : 20 mars 2019

Pagination : $53-73$

ISBN : 978-2-7535-7782-4

ISSN : 0399-0826

\section{Référence électronique}

Aurélie Hess et Philippe Hrodĕj, « Les marins du Plessis-Bertrand à l'époque de Louis XIv : les opportunités d'une base de données », Annales de Bretagne et des Pays de l'Ouest [En ligne], 126-1 | 2019, mis en ligne le 01 janvier 2022, consulté le 06 janvier 2022. URL : http:// journals.openedition.org/abpo/4358; DOI : https://doi.org/10.4000/abpo.4358 


\title{
Les marins du Plessis-Bertrand à l'époque de Louis XIV : les opportunités d'une base de données
}

\author{
Aurélie Hess \\ Ingénieur d'étude CNRS, université de Bretagne sud \\ Philippe HRODĔJ \\ Maître de conférences en histoire moderne, université de Bretagne sud
}

La constitution d'une base de données concernant les gens de mer est toujours une œuvre de longue haleine. On aura beau dire que la France d'Ancien Régime n'a jamais obtenu le nombre de marins qui lui était nécessaire (le système des classes mis en place par Colbert n'en fournit que 50 à 60000 ), la masse documentaire que génère cet amour précoce pour la bureaucratie est une source d'archives que tous les pays qui ont un littoral lui envient aujourd'hui. La base H3D2 ${ }^{1}$, dont l'objet est de recenser les marins du Plessis-Bertrand (territoire regroupant les paroisses de Cancale, Paramé, Saint-Coulomb, Saint-Ideuc, Saint-Méloir), n'échappe pas à ce paradoxe : malgré la perte de sources importantes comme les papiers de l'amirauté de Brest ou certains rôles d'équipage, ce qui reste ne manque pas d'impressionner pour qui veut s'y plonger.

La base en elle-même a vocation à s'étendre à l'ensemble du XVIII siècle et, une fois son efficience constatée, à être inclue dans un projet qui empruntera moins à la chose intergalactique et s'intitulera "Gens de mer des temps modernes : carrières maritimes, attaches littorales et état sanitaire ". Seront alors ajoutés deux champs supplémentaires qui, comme le titre l'indique, porteront sur l'aspect sanitaire avec un volet épidémiologique, un volet anthropologique et ethnologique, et sur l'environnement socio-économique à proprement parler, à savoir les comportements fami-

1. Cette base n'aurait pu être créée sans la participation d'érudits locaux, de généreux et acharnés chercheurs, en l'occurrence Rolland Delépine, Thierry Huck et, particulièrement dans le cadre de cet article, Aristide Delarose qui nous a fourni ses précieuses fiches généalogiques, fruit de nombreuses années de compilation, tirées des registres dits BMS de Cancale (baptêmes, mariages et sépultures). 
liaux et démographiques. Cette base est destinée, à terme, à s'ouvrir, si ce n'est à tous (il y aura, quoi qu'il en soit, une partie publique), à ceux qui en feront la demande et, naturellement, au monde de la recherche. Pour ce qui est de s'en servir comme modèle, il est imaginable d'introduire le principe d'un cahier des charges. Les modalités concernant le support, le lieu de stockage et la date d'ouverture seront définies par la suite.

Mais comme il faut un début à tout, cette base a été construite selon un cahier des charges où la préoccupation première a été que l'enregistrement des données obéisse à certaines conditions. Ceux qui figurent dans ce corpus doivent tous apparaître dans les registres des matricules (ceux qui vont de 1697 à 1731) conservés au Service Historique des Armées (SHD) de Brest. En outre, ils doivent tous avoir navigué entre 1697 et 1702, période de paix très ténue séparant la guerre de la Ligue d'Augsbourg de celle de la Succession d'Espagne. N'ont pas été retenus ceux dont on n'a pas trouvé cette preuve d'un embarquement ou qui, mousses bien souvent, n'ont pu être augmentés, c'est-à-dire intégrés aux classes, simplement parce qu'ils ont disparu auparavant. Le choix du verbe disparaître étant susceptible d'englober plusieurs raisons même si le décès reste la cause la plus plausible. Enfin, pour mieux mesurer l'impact de la guerre sur cette population, ils doivent tous être vivants au début de l'année 1702, la guerre étant déclarée à l'Angleterre et aux Provinces-Unies au mois d'avril. Si tous les marins décédés en 1702 sont pris en compte, ceux que le signalement donne pour morts en 1701 ne le sont pas.

Le croisement de ces sources ouvre de multiples champs d'investigation et permet de découvrir non seulement des gens de mer, dont plus de la moitié sont des Cancalais, mais également leur famille, leur environnement social à terre comme à bord, à travers les rôles d'équipage, notamment lors d'embarquements pour Terre-Neuve. Il permet encore de mieux cerner les choix de carrière qui se font selon la conjoncture et les opportunités qu'elle offre, l'impact de la guerre sur cette population, la mortalité en mer et, quelque part, l'ouverture au monde très précoce de ces marins, admirables à bien des égards et dignes du plus grand respect.

\section{Exploitation de la base à partir d'un échantillon du corpus : les lettres $\mathbf{G}$ et $\mathrm{H}$ des marins de Cancale}

\section{Un marin pour 21 personnes ${ }^{2}$}

L'exploitation des registres de baptêmes, mariages, sépultures (BMS) de la paroisse de Cancale disponibles en ligne aux Archives départementales d'Ille-et-Vilaine, les registres de matricules et les rôles d'équipage conservés aux Services Historiques de la Défense ou dans les fonds d'amirauté des Archives départementales, ont permis de mettre en place une base

2. Les individus issus d'alliances spirituelles seront inclus par la suite. 
de données qui regroupe l'ensemble des informations recueillies dans ces trois sources.

Ces dernières donnent accès à un certain nombre d'informations sur l'ensemble des individus du corpus et sur les personnes qui leur sont liées (parents, enfants, grands-parents, frères et sœurs, oncles et tantes, leurs conjoints et enfants...). Une première table recense tous les individus ainsi identifiés. Alors même que seuls les marins dont le nom de famille commence par les lettres $\mathrm{G}$ et $\mathrm{H}$ de la paroisse de Cancale représentent 111 marins du corpus (soit 12 \%), la base regroupe déjà des informations pour 2547 individus. Le travail de dépouillement des BMS réalisé par Aristide Delarose a donc permis de recueillir des renseignements relatifs à 2436 personnes qui ont un lien familial avec ces 111 marins. Elles se répartissent en 1241 femmes, 1195 hommes dont 54 sont des marins du corpus pour lesquels le nom commence par une autre lettre que le $\mathrm{G}$ ou le $\mathrm{H}$.

Pour chaque personne identifiée, le tableau 1 synthétise les informations individuelles reprises.

Tableau 1 : information individuelle

\begin{tabular}{|l|l|}
\hline & \multicolumn{1}{|c|}{ Sources } \\
\hline Nom & BMS \\
\hline Prénom & BMS \\
\hline Sexe & BMS \\
\hline Marin & $\begin{array}{l}\text { Registre des matricules } \\
\text { Pour les marins du corpus. } \\
\text { BMS }\end{array}$ \\
\hline Date de naissance & BMS \\
\hline Lieu de naissance & BMS \\
\hline Date de décès & $\begin{array}{l}\text { BMS } \\
\text { Registre des matricules }\end{array}$ \\
\hline Lieu de décès & $\begin{array}{l}\text { BMS } \\
\text { Registre des matricules }\end{array}$ \\
\hline Causes du décès & $\begin{array}{l}\text { BMS } \\
\text { Registre des matricules } \\
\text { Rapports de mer }\end{array}$ \\
\hline Taille & $\begin{array}{l}\text { Registre des matricules } \\
\text { et rôles d'équipage }\end{array}$ \\
\hline Poil & $\begin{array}{l}\text { Registre des matricules } \\
\text { et rôles d'équipage }\end{array}$ \\
\hline Lieu de résidence & Registre des matricules \\
\hline Commentaires & $\begin{array}{l}\text { Registre des matricules, } \\
\text { parfois rôles d'équipage } \\
\text { Rapports de mer }\end{array}$ \\
\hline
\end{tabular}


Le lien est fait entre les individus à partir des femmes qui sont mères et épouses. Les liens entre père et enfants ou entre frères et sœurs mais aussi entre les oncles, tantes et neveux peuvent ainsi être mis en évidence. Une analyse des réseaux de parenté peut donc être réalisée. Dans cette table des relations, la date de mariage est aussi indiquée.

Les registres des matricules des marins du corpus donnent accès à l'ensemble des services des marins, que ce soit sur des vaisseaux du roi ou sur des vaisseaux de particuliers pour la course ou pour la pêche (cf. image 1). "Ils sont remis à jour chaque année lors des revues de quartier par l'officier des classes assisté du syndic des gens de mer. Ils notent les mariages, les changements d'adresse, les maladies, les décès $[\ldots]^{3}$ ". Ainsi la base va pouvoir recenser l'ensemble des campagnes des marins du corpus et mettre en évidence tout ce qui les relie (proximité géographique, coïncidence des embarquements, liens familiaux). Le croisement de ces informations avec celles recueillies à partir des rôles d'équipage permet de compléter cette approche en recherchant tous les marins venant du PlessisBertrand (Cancale, Saint-Méloir, Saint-Ideuc, Paramé, Saint-Coulomb), qu'ils soient ou non dans le corpus initial.

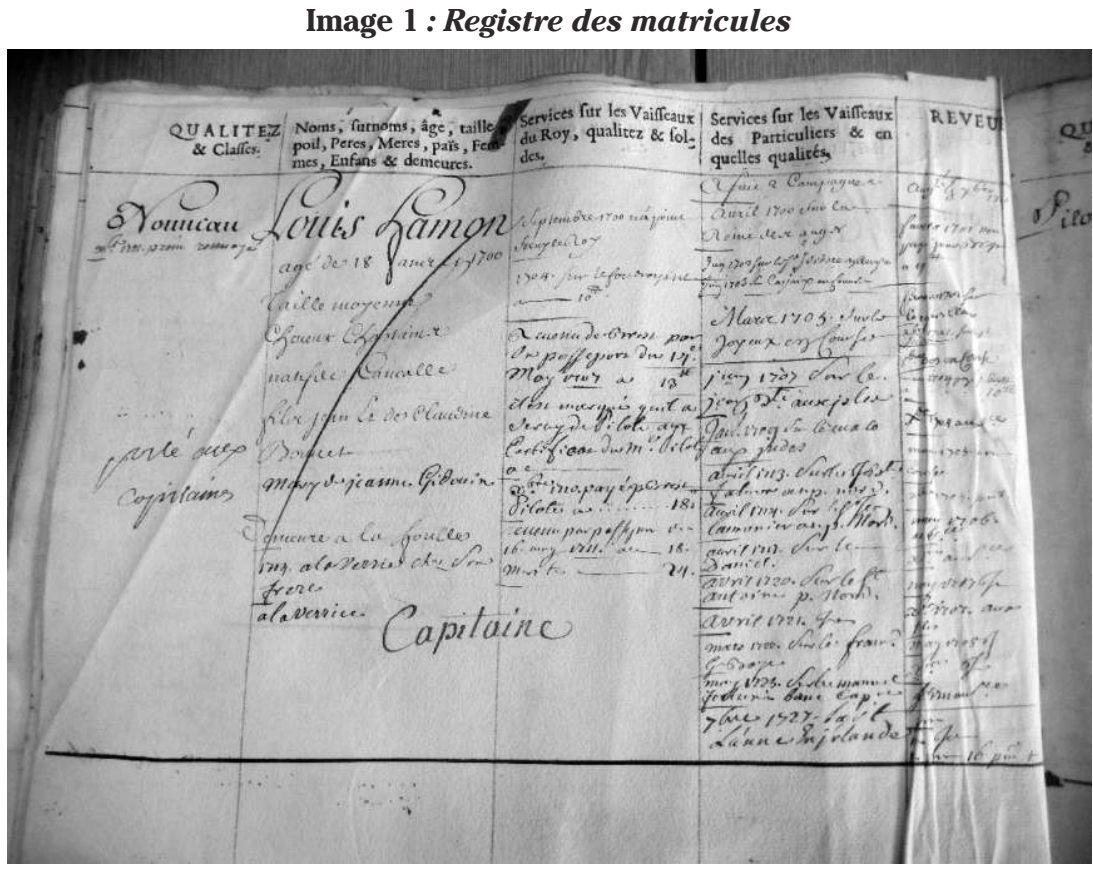

(Service Historique de la Défense (SHD), Brest, 1P3-50)L'étude des navigations accomplies par les marins " $\mathrm{G}$ et $\mathrm{H}$ " du corpus représente plus de

3. LE BOUËDEC, Gérard, Les Bretons sur les mers, Rennes, Ouest-France, 1999, p. 228. 
mille campagnes recensées à partir du dépouillement des registres des matricules (1097 exactement). Les rôles d'équipage permettent d'affiner l'information disponible comme le montre le tableau 2.

Tableau 2 : Informations sur les embarquements

\begin{tabular}{|l|l|}
\hline & \multicolumn{1}{|c|}{ Source } \\
\hline Nom du vaisseau & $\begin{array}{l}\text { Registre des matricules } \\
\text { Rôles d'équipage }\end{array}$ \\
\hline Date de l'appareillage & Rapports de mer \\
\hline Port appareillage & Rôles d'équipage \\
\hline Date du retour & Rapports de mer \\
\hline Type de campagne & $\begin{array}{l}\text { Registre de matricules } \\
\text { Rôles d'équipage } \\
\text { Rapports de mer }\end{array}$ \\
\hline Nom du capitaine & Rôles d'équipage \\
\hline Tonnage du vaisseau & Rôles d'équipage \\
\hline Nombre de canons & Rôles d'équipage \\
\hline Nombre d'hommes d'équipage & Rôles d'équipage \\
\hline Destination & $\begin{array}{l}\text { Rôles d'équipage } \\
\text { Rapports de mer }\end{array}$ \\
\hline
\end{tabular}

Compte tenu des hypothèses de constitution du corpus (cf. supra), les marins pris en compte sont nés entre 1628 pour le plus âgé et 1687 pour le plus jeune. L'année de naissance des 111 marins du corpus est restituée dans le graphique suivant.

\section{La guerre et sa moisson}

Une étude sera effectuée pour définir les critères qui font que le commissaire aux classes décide de "rayer " un marin des matricules ainsi que le calcul de "l'âge de la retraite ". Le faible nombre de quinquagénaires s'explique par le décès avant 1702 de beaucoup de ceux de cette classe d'âge, mais aussi parce que, vieux, " pourris " comme cela apparaît parfois, handicapés, invalides ou malades, les autres ont été rayés des classes, ce qui ne signifie pas forcément la fin de leurs activités maritimes : ils sont simplement inaptes à embarquer sur les vaisseaux du Roi. L'âge au moment du décès peut dans la majeure partie des cas être reconstitué.

Au sein de cet échantillon, une fois ôtés ceux dont on ne connaît pas les circonstances du décès ${ }^{4}, 36$ sont morts en mer (ou sur une grève de Terre-Neuve), 8 en prison, soit 54 \% ; les 36 autres n'ont pas forcément évité la malemort, car on ne peut échapper au risque de noyade lors de la pêche côtière ou d'accidents qui ne seraient pas portés à la connaissance du com-

4. Il suffit que ces marins soient décédés à l'hôtel-Dieu de Saint-Malo, les registres de sépulture ne commençant qu'en 1730, ils demeurent invisibles. 
missaire. Celui-ci, une fois le marin rayé des classes, se montre bien moins circonspect et indiquer en lettres majuscules MORT au bas de la matricule ne signifie pas grand-chose pour un historien, surtout 300 ans après. Si 36 marins décèdent à Cancale, un au moins finit ses jours sous d'autres cieux. Eustache Gourdel, fils d'Eustache et Suzanne Renard meurt vers 1735 à Québec où il s'était établi depuis 1717. Il avait épousé en 1700 à Cancale Thomasse Marguerite Hamon avec laquelle il avait eu deux enfants décédés dès leur première année de vie ${ }^{5}$. Veuf en 1710 , il aurait franchi le pas au moment où la France se donne une nouvelle forteresse sur l'île du CapBreton (Louisbourg) pour protéger l'embouchure du Saint-Laurent après la perte du contrôle de Terre-Neuve et de l'Acadie orientale. Au Québec il se serait remarié et aurait eu sept enfants selon le registre des matricules.

\section{Graphique 1 : Répartition des marins suivant leur année de naissance}

\begin{tabular}{|c|c|}
\hline Année & Nombre de marins \\
\hline$\leq 1650$ & 6 \\
\hline$] 1651-1660]$ & 23 \\
\hline$] 1661-1670]$ & 28 \\
\hline$] 1671-1680]$ & 42 \\
\hline$>1680$ & 12 \\
\hline Total & 111 \\
\hline
\end{tabular}

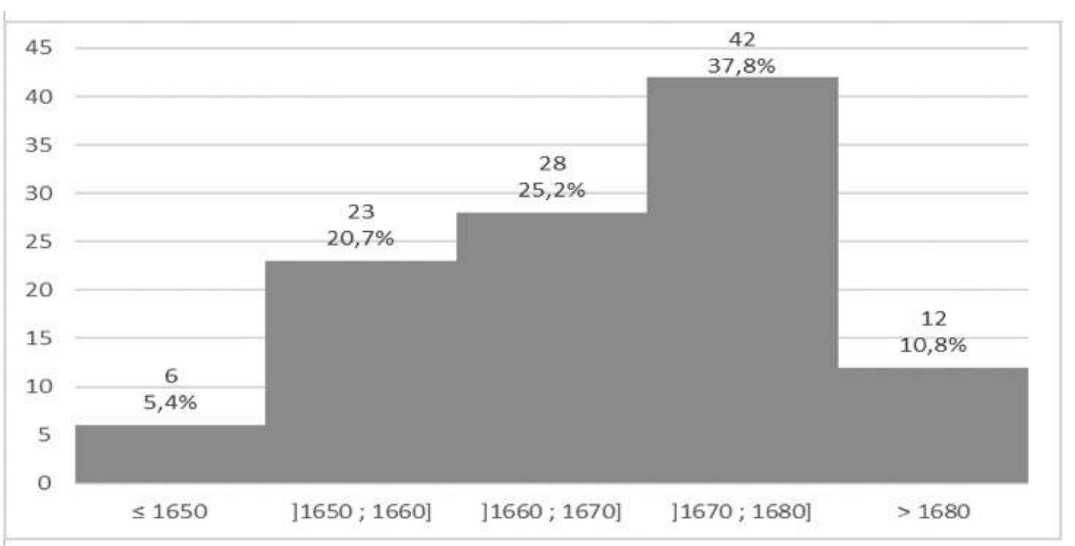

Quel impact a pu avoir la guerre de Succession d'Espagne sur cet échantillon? En moyenne, les marins décèdent à 51 ans. Mais parmi eux, 8 (soit 10 \%) sont décédés en prison en Angleterre ou en Irlande. Jacques Gauvain et Jacques Gervin étaient embarqués en 1703 sur le Hasardeux au départ

5. Arch. dép. d'Ille-et-Vilaine, registres paroissiaux et états civils en ligne. Nous utilisons le dépouillement effectué par Aristide Delarose. 
du Port-Louis pour la course lorsque leur bateau a été pris par les Anglais. Ils sont morts dans les prisons anglaises en 1704. Trois marins du corpus, Pierre Gaultier, Bertrand Gaultier et Pierre Girard sont aussi embarqués ensemble sur le Desmarais pour la course et sont faits prisonniers en Angleterre où deux d'entre eux décèdent respectivement à 26 et 43 ans ; le dernier marin, Pierre Gaultier, reviendra en France où il fera encore huit campagnes jusqu'en 1724. Plus chanceux, il décèdera à Cancale en 1731, à l'âge de 66 ans. En 1705, Olivier Guillou meurt dans les prisons irlandaises après que le corsaire la Rieuse ait été capturé : l'occasion de souligner la très forte mortalité des Malouins dans les geôles britanniques où le temps plus long de l'échange et les privations sont un facteur aggravant, comparativement à ce que l'on observe aux Provinces-Unies. Sur les 36 Cancalais décédés en mer, 20 disparaissent lors d'une campagne à Terre-Neuve, 7 à bord d'un corsaire. Deux marins sont morts sur un vaisseau du roi à Brest. Ce sont donc au moins 17 hommes qui meurent directement à cause de la guerre, soit $21 \%$. Cancale, comme les autres ports, a payé un lourd tribut à ce conflit et la course est une activité particulièrement mortifère.

\section{Le mariage, marqueur social et bonne engeance}

La plupart de ces hommes se marient. Il ne faut pas oublier qu'être marié est un marqueur social qui fournit à celui qui a convolé en justes noces une stabilité appréciée des armateurs et autres bourgeois.

Tableau 3 : Situation matrimoniale des marins du corpus

\begin{tabular}{|l|c|c|}
\hline & Nombre de marins & $\%$ \\
\hline Célibataires & 19 & 17 \\
\hline 1 épouse & 80 & 72 \\
\hline 2 épouses & 10 & 9 \\
\hline 3 épouses & 2 & 2 \\
\hline Total & 111 & 100 \\
\hline
\end{tabular}

L'analyse de la situation matrimoniale des 111 marins montre (tableau 3) que presque un marin sur cinq demeure célibataire. Ces hommes célibataires décèdent en moyenne un peu avant leur trente et unième année sans avoir pu se marier et fonder une famille. On sait aussi que, dans quelques cas de blessures invalidantes ou de handicap, certains ne se marieront jamais. En fait, l'analyse montre que l'âge moyen au premier mariage est de presque 28 ans. Les marins célibataires ont une espérance de vie d'un peu plus de 30 ans, inférieure de 20 ans à l'espérance moyenne des marins du corpus. Seul, Étienne Gervin est décédé à 56 ans tout en ne s'étant jamais marié. En mars 1697, à l'âge de 42 ans, il est " tombé d'un mal et est paralytique $^{6}$ ", ce qui évoque une crise d'épilepsie ou une crise de folie. Dans

6. Service historique de la Défense de Brest, matricules, 1P3 11. 
les registres de matricules, ce marin est souvent qualifié de fou montrant par-là que son mal a empiré avec le temps : on dit encore " qu'il a viré d'esprit et qu'il court dans les champs ${ }^{7}$ ". Augmenté en 1683, ce matelot de la troisième levée ne semble avoir embarqué sur un vaisseau du roi qu'en 1684, à l'époque l'Apollon, avec 10 livres de solde mensuelle. Il est bien levé à nouveau en 1692 mais sans précision. Son état a-t-il un lien quelconque avec sa solde? Celle-ci a été remontée à 15 livres. Il ne paraît plus naviguer après 1699 et est rayé des classes en 1705. Ce " haut mal " est bien un motif de célibat après 30 ans.

Les 106 épouses des marins ont en moyenne 25,5 ans au moment de leur mariage, elles sont donc un plus jeunes, de trois ans, que leur époux. Seules trois femmes sont plus âgées. Jacquemine Robinot, veuve de Macé Legallais de la Bretonnière, décédé en service à Brest en décembre 1689, est mère d'une fille Anne, née en 1685; elle s'est remariée en 1695 à l'âge 40 ans avec Jean Gourdel des Longs Prés alors âgé de 24 ans. De cette union ne naît aucun enfant, et c'est encore un mariage étrange; Jacquemine décède l'année suivante et quelques mois plus tard, Jean Gourdel épouse Gillette Lhostellier, dont il aura sept enfants. Trois d'entre eux au moins laisseront une postérité avérée. Nicole Dagosne, âgée de 38 ans, veuve depuis 14 ans et mère de deux enfants, épouse en secondes noces Laurent Grossin des Saudrais lui-même veuf et père de 12 enfants. Ils auront ensemble deux autres enfants. Enfin, Julienne Laisné épouse, alors qu'elle a 38 ans, Étienne Hardy qui est son cadet de 12 ans. De cette union naîtra un seul enfant, un garçon, qui ne survivra pas.

Ce sont donc 83 \% des hommes qui se marient une ou plusieurs fois. La naissance du premier enfant, ce qui définit l'intervalle protogénésique, a lieu en moyenne 18 mois après le mariage. L'intervalle intergénésique est de trois ans, une moyenne exacte de 1086 jours. Le nombre d'enfants par marin marié est de $5,7^{8}$. Ces couples ont autant de filles que de garçons (261 filles contre 260 garçons). Dans ces sources, il n'est fait mention que de 34 enfants qui sont eux-mêmes marins comme leur père. Dix d'entre eux sont intégrés au corpus.

L'espérance de vie des enfants des marins du corpus est de 35 ans. On remarque une mortalité infantile apparente très forte (cf. tableau 4). En effet, 10,7 \% au moins des enfants décèdent durant les deux premières années de vie ${ }^{9}$. Mais ce sont quelque 300 individus dont on ignore l'âge de décès. Il est aisé d'imaginer que la connaissance de cette information ren-

7. Service historique de la Défense de Brest, matricules, 1P3 3. Nous remercions Thierry Huck pour le traitement de ce registre.

8. Cabantous, Alain, Dix mille marins face à l'océan, Paris, Publisud, 1991, p. 349. On est donc très éloigné de ce qui a été trouvé à Dunkerque en 1695, soit 1,39 enfant par famille de gens de mer.

9. Mais il sera très difficile ici d'avoir des certitudes; si le jour de naissance et celui du baptême sont indiqués, le décès d'enfant n'est pas systématiquement inscrit sur les registres de sépulture, loin s'en faut. 
forcerait considérablement la part de la mortalité infantile et juvénile. Dans l'absolu, en considérant que ceux dont la date de décès reste inconnue ont tous moins de 20 ans, mortalité infantile et mortalité juvénile s'élèveraient à plus de $72 \%$; au moins un enfant ou adolescent sur deux meurt avant l'âge adulte, ce qui est habituel, et, plus vraisemblablement, deux sur trois. Après 20 ans, la forte mortalité des gens de mer se vérifie, 17 \% des hommes décèdent dans la fleur de l'âge entre 20 et 40 ans contrairement aux femmes (3\%).

Tableau 4 : Âge au décès des enfants des marins du corpus

\begin{tabular}{|l|c|c|c|}
\hline \multicolumn{1}{|c|}{ Âge au décès } & \multicolumn{3}{c|}{ Nombre d'enfants des marins } \\
\hline & Filles & Garçons & Total \\
\hline Non renseigné & 166 & 136 & 302 \\
\hline$[0 ; 2]$ ans & 26 & 30 & 56 \\
\hline$] 3 ; 10]$ ans & 5 & 4 & 9 \\
\hline$] 11 ; 20]$ ans & 3 & 8 & 11 \\
\hline$] 21 ; 40]$ ans & 8 & 44 & 52 \\
\hline$] 41 ; 60]$ ans & 19 & 18 & 37 \\
\hline$>60$ ans & 34 & 20 & 54 \\
\hline & 261 & 260 & 521 \\
\hline
\end{tabular}

\section{Matelot comme ton père}

Pour les 111 marins déjà étudiés dans le corpus, 85 mères sont identifiées. Certaines sont la mère d'un seul marin ou de deux à quatre marins du corpus (cf. tableau 5).

\section{Tableau 5 : Nombre de marins du corpus par mère}

\begin{tabular}{|c|c|}
\hline $\begin{array}{c}\text { Nombre d'enfants marins } \\
\text { retenus dans la base }\end{array}$ & Nombre de mères \\
\hline 1 & 66 \\
\hline 2 & 14 \\
\hline 3 & 3 \\
\hline 4 & 2 \\
\hline Total & 85 \\
\hline
\end{tabular}

Ces 85 femmes sont mères de 495 enfants, soit 5,82 enfants par mère. Une sur-représentation des enfants de sexe masculin est observée, en effet $62 \%$ sont des garçons soit deux enfants sur trois. Pour 120 d'entre eux, il est fait mention d'une activité maritime. Le nombre d'enfants de sexe féminin est de 188. L'étude des registres paroissiaux (BMS) montre que parmi ces filles, 51 sont mariées. Pour 25 d'entre elles, soit une femme mariée 
sur deux, l'époux est marin. Une recherche sur les époux et leur qualité haussera certainement cette donnée.

Ces remarques en entraînent d'autres. Pour mieux cerner l'environnement " marin " et cette chaîne de transmission de la "vocation " des gens de mer, une étude a pu être menée réunissant pour chaque homme du corpus la profession du père, du grand-père maternel et du beau-père (ou des beaux-pères). La lettre $\mathrm{G}$ a été particulièrement visée et a fourni un corpus de 203 hommes de ces trois catégories. La première constatation est qu'une limitation liée à la date de formation des classes oblige à retirer quelque 55 hommes, décédés ou incapables de naviguer avant le premier rôle de 1670 et dont aucune source ne vient indiquer la situation. Par ailleurs, 21 hommes sont purement et simplement inconnus. Difficile également d'identifier ceux exerçant une autre profession. Pierre Gaultier (1642-1716) est dit maître cordonnier. Pourtant son père Paul (1601-1653) est marin, tout comme ses propres fils, Josselin, mort à l'hôpital de Rochefort en 1695 et Pierre (inclus dans le corpus) qui cumule une vingtaine de campagnes terre-neuviennes, six embarquements en course et autant de services du roi quand, après 1724, il ne se livre plus qu'à la pêche au poisson frais. D'ailleurs rien ne permet d'être sûr que maître Gaultier ait embarqué. Alain Hamon (1619-1690), mort à Saint-Coulomb, est qualifié de laboureur. Ses deux fils sont gens de mer (deux François du corpus). Aux côtés de ces individus bénéficiant d'une mention, 27 n'ont été trouvés nulle part (listes de 1670, 1688, 1697, 1731). Le résultat est néanmoins édifiant pour ce bourg accroché à la côte, aux terres peu fertiles et peu étendues.

\section{Tableau 6 : Profession des pères, beaux-pères et grands-pères des 72 marins du corpus}

\begin{tabular}{|c|c|c|c|c|c|c|}
\hline Inconnus & $\begin{array}{c}\text { Décédés } \\
\text { avant 1670 }\end{array}$ & $\begin{array}{c}\text { Autre } \\
\text { profession } \\
\text { (a) }\end{array}$ & $\begin{array}{c}\text { Indéterminés } \\
\text { (b) }\end{array}$ & $\begin{array}{c}\text { Gens de } \\
\text { mer avérés } \\
\text { (c) }\end{array}$ & $\begin{array}{c}\text { Total (a) + } \\
\text { (b) + (c) }\end{array}$ & $\%$ \\
\hline 21 & 55 & 1 & 27 & 99 & 127 & 78 \\
\hline
\end{tabular}

On voit bien que, quel que soit l'angle choisi, l'incertitude domine. En réalité, on se trouve à une époque où les archives sont relativement bavardes mais seulement pour la génération de 1680 et les suivantes. En revanche, l'emploi des hommes comme marins, hors la liste des inscrits de 1670 , demeure très flou avant $1682^{10}$. Ce qui est certain c'est qu'au moins 78 \% de l'encadrement de nos gens de mer est de la même veine, du même tonneau. Les travaux de Thierry Huck ont montré à quel point le nombre réel de pères marins est infiniment supérieur à Cancale. À partir des matri-

10. Voir le site créé et entretenu par Aristide Delarose, Cancale et à l'entour. Territoire \& Histoire. Cette liste (Rôle général des officiers mariniers et matelots de la lieutenance du Roi de Monsieur le marquis de Coëtlogon, évêché de Saint-Malo et Dol) est la seule sur laquelle on puisse réellement se fonder avant 1682 où commencent les matricules. [http://adelarose.free.fr/] 
cules des mousses ouverts en 1729, 241 mousses ont massivement des matelots pour père (180 soit $72 \%$ ), mais ce chiffre, en comptant la maistrance et quatre pêcheurs, s'élève à 196 pères marins soit $78 \%{ }^{11}$. Reste à établir s'il s'agit d'un particularisme lié à la position même de Cancale. À Saint-Coulomb, ce taux, pour être plus bas, n'en reste pas moins élevé. Il faut naturellement mieux cerner le statut de ces hommes, "le profil de pêcheur paysan et/ou artisan ", sans douter que "la pluriactivité reste une nécessité extrêmement fréquente ${ }^{12}$ ".

Dernière chose et qui n'a rien d'une curiosité, le moment du mariage. À l'exception du mois de décembre, et même si les mois de juin à septembre sont moins propices, liés à la période habituelle de navigation à destination de Terre-Neuve, rien ne démontre un interdit quelconque. Le dépouillement des matricules indique d'ailleurs que, bien souvent, tel ou tel n'embarque pas au loin, ce qui se vérifie par le fait d'être présent lors des revues ou avec la remarque " est resté au pays ", souvent pour cause de maladie. On ne parlera pas d'années sabbatiques, mais de pause, d'interruptions souvent contraintes. Bien entendu, endogamie, homogamie et tout ce qui a trait à la consanguinité se mesure assez facilement mais demandent à ce que la base de données soit complétée.

\section{Graphique 2 : Nombre de mariages selon le mois de l'année des marins du corpus}

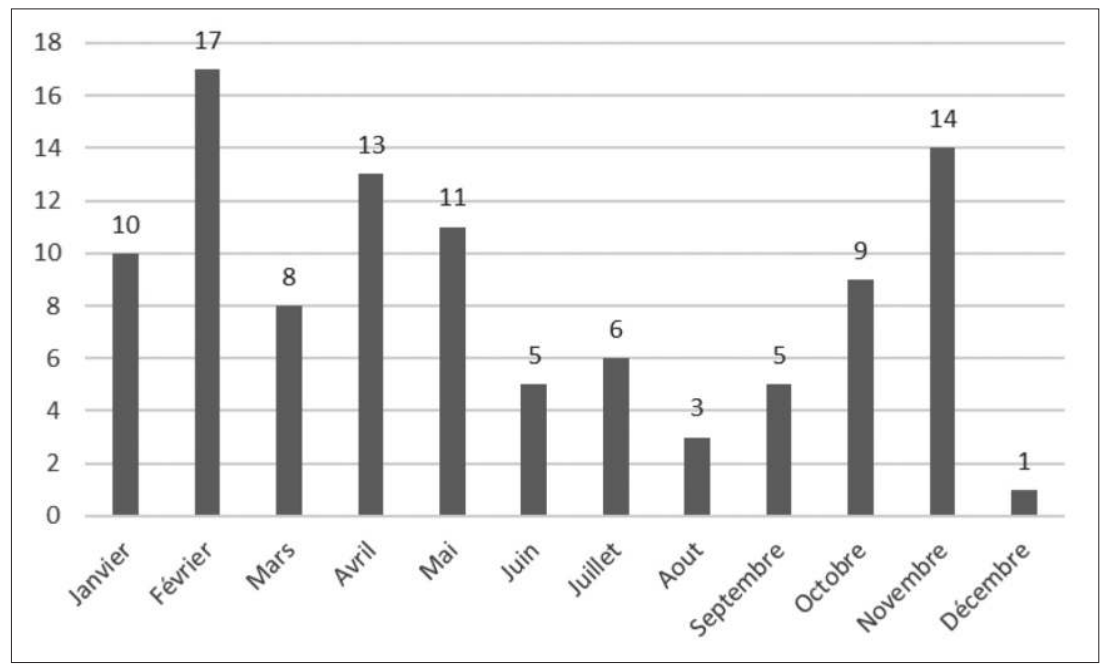

11. Huck, Thierry, dépouillement des séries 1P3 51 à 55. État de service des mousses de 1729 à 1786, Service historique de la Défense de Brest.

12. Buti, Gilbert et CaBantous, Alain, Etre marin en Europe occidentale 1550-1850, Rennes, Presses universitaires de Rennes, 2016, p. 22. Voir également Charpentier, Emmanuelle, Le littoral et les hommes. Espaces et sociétés des côtes nord de la Bretagne au XVIII siècle, Rennes, Presses universitaires de Rennes, 2013. 
De la même façon, les naissances couvrent tous les mois de l'année, avec une prédominance pour la période allant de septembre à décembre, soit une conception allant de décembre à mars. Rien de surprenant. Faut-il y voir une raison d'un fort taux de mortalité infantile dû à la froidure et à l'humidité " conditions atmosphériques propices à des contagions particulièrement redoutables pour de jeunes existences ${ }^{13}$ " ? Ce sera encore un point à vérifier, tout comme sera à mesurer l'âge où il arrive que les enfants " survivants " deviennent orphelins, le plus souvent de père.

\section{Graphique 3 : Nombre de naissances des enfants des marins du corpus} selon le mois de l'année

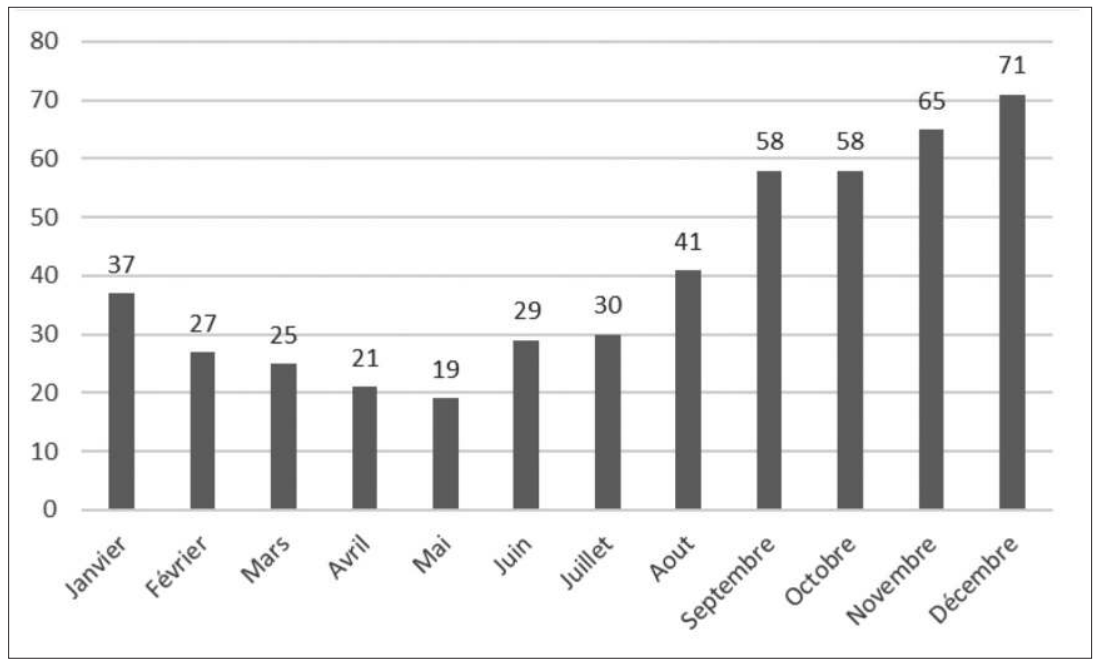

\section{Embarquements et environnements familial et géographique}

Une fois constituée, la base H3D2, ayant en mémoire le contenu des registres des matricules, des rôles d'équipage et des informations issues des registres de BMS, sera en mesure de fournir immédiatement tout ce qui concerne l'environnement familial et géographique lié à tel ou tel individu. Elle sera tout autant susceptible de répondre à des questions portant sur la nature des embarquements et les événements liés à toutes les navigations. Ainsi plusieurs centaines, voire plusieurs milliers, de trajectoires a priori différentes se croiseront automatiquement et fourniront autant de combinaisons intéressantes. D'évidence, ce travail de recomposition est tout simplement impossible sur fiche au-delà de quelques dizaines d'individus. Prendre un exemple ne manque pas d'intérêt, notamment pour imaginer quelles requêtes pourront être formulées.

13. Cabantous, Alain, Dix mille marins..., op. cit., p. 353. 
L'idée première a été de choisir un marin dont la matricule était incomplète, un cas particulier dans la mesure où, durant toute sa carrière, cet homme s'est vu attribuer, tant dans le rôle des classes que sur les rôles d'équipage un âge qui ne lui correspondait pas et qui le rajeunissait de huit ans. Cet écart est assez rare et explique largement que Josselin Grossin n'ait été " augmenté " qu'en 1708 alors qu'il naviguait depuis au moins 1698. Il a donc été très longtemps enregistré comme mousse sans qu'une explication se détache plus qu'une autre pour cette particularité ${ }^{14}$. S'agissait-il de le protéger en retardant l'année où il devrait servir le roi? Cela se vérifie lorsque le marin se trouve chargé d'une famille particulièrement nécessiteuse, sur intervention bien souvent du recteur ou de la communauté. Rien n'interdit ici cette interprétation; il y en a sans doute d'autres. Le père, Gilles, est décédé en 1696/1697 à 49 ans; il était embarqué comme matelot sur la frégate corsaire le Saint-Antoine, de 600 tonneaux et 52 canons, et l'on perd sa trace sur une prise qui n'est jamais arrivée à bon port. La guerre de la Ligue d'Augsbourg ne s'est pas arrêtée à ce prélèvement. Le cousin de Josselin, Jean, est décédé en 1692 à La Hougue, peu après avoir quitté le Soleil-Royal. Son oncle Philippe n'a pas eu ce sursis, " tué " "brûlé " sur ledit vaisseau ${ }^{15}$. Un autre cousin, Julien, est mort sur la même frégate que son père ${ }^{16}$. De la famille qui habite le Haut Bout, - on sous-entend le bout du territoire de Cancale, la partie la plus proche de la pointe du Grouin -, ne reste que Jacques, un oncle " très incommodé " qui a été rayé des classes. Non loin, se trouvent deux cousins éloignés. De ces deux frères vivant à la Mancelière et à la Ville-es-Gris, on retiendra que la guerre ne les a pas plus épargnés car ils ont perdu deux autres frères : Olivier sur la Licorne en 1691 et Marc qui meurt peu après, en 1698, sur la Charmante. Jean, âgé de 28 ans, a enchaîné les embarquements corsaires durant le conflit. Il se trouve sur le Renau en 1697, revenant des Antilles ${ }^{17}$. Son aîné de cinq ans, Gilles, était embarqué sur l'Hercule en 1696, il est parti au Canada sur le Phélypeaux. Ce tour d'horizon pourrait conforter l'idée d'une situation d'isolement tragique. De ce que l'on peut reconstituer des hommes de la famille Grossin, la guerre en a moissonné une partie, laissé les autres dans un piètre état, et ceux qui demeurent indemnes sont au loin.

L'un des objectifs donnés à la base est donc d'aller plus loin. La situation familiale touche forcément les collatéraux maternels. Il y a au Haut Bout un dénommé Robert Grossin. On ne trouve pas de lien de parenté visible qui se puisse établir avec Josselin. En fait, la mère de Josselin, Hélène Cliquin, est la sœur de Jeanne et Robert a épousé Jeanne en 1684, dont il a eu à cette époque six enfants. Il a certes été blessé au pied sur le Conquérant,

14. Service historique de la Défense de Brest, matricules 1P3 17.

15. Huck, Thierry, "La tragédie du Soleil Royal ", Les Cahiers de la vie à Cancale, 2008, $\mathrm{n}^{\circ} 32$, p. 57-61. Embrasement et explosion, ce sont 22 Cancalais et 5 Colombanais qui disparaissent dans cette tragédie.

16. Service historique de la Défense de Brest, matricules 1P3 3.

17. Campagne de Renau d'Eliçagaray aux Antilles (HRODĔJ, Philippe, L'amiral Du Casse, l'élévation d'un Gascon sous Louis XIV, Paris, Librairie de l'Inde, 1999, p. 197 et sq.). 
vaisseau du roi, en 1692. Le commissaire aux classes indique même qu'il s'est déplacé en 1694 pour vérifier si la blessure était toujours invalidante ${ }^{18}$. Il semble que oui : Robert est déclaré estropié en 1697, présent au pays. En 1705 , il est dit qu'il " est non propre au service ${ }^{19}$ ". Cela ne l'empêche pas de repartir en mer. Dès 1698, il embarque sur la Faluère à destination du Petit Nord. Et puis Hélène et Jeanne ont des frères. Gilles, qui a 41 ans en 1697, n'est pas non plus en grande forme. Présent au pays durant les années 1694 à 1696, il est déclaré malade en 1697. L'année suivante, il embarque également sur la Faluère mais en mai 1699, on le retrouve au lit, étant " très mal " et le commissaire peut confier " qu'il est tout pourri, ce que j'ai vu est certifié. [II] est digne de pitié, joint le certificat du médecin et du chirurgien dudit lieu ${ }^{20}$ ". Il n'a pourtant pas fini d'aller à Terre-Neuve ni de servir le roi : on le retrouvera sur le Henry (Serquigny) à Velez Malaga, puis en 1705 sur le Mercure (La Rochalard). Gilles Cliquin vit au Haut de Cancale, comme Robert Cliquin, autre oncle de Josselin. Robert sert le Roi en 1697 sans que l'on sache dans quelles conditions. C'est un canonnier qui est soldé 15 livres par mois. En 1698, lui aussi monte sur la Faluère, comme pêcheur. Que s'est-il passé durant ce voyage? En 1699, le commissaire lui rend visite : " vu, écrit ce dernier, ayant reçu le bon Dieu et hors d'état de servir, étant si diminué qu'il ne paraît [être] rien du tout ${ }^{21}$ ". À 37 ans, il repartira bientôt, sa carrière maritime commencée en 1683 est loin d'être achevée. Reste Olivier, autre frère, qui vit au Haut Bout. Pour lors il est à Plaisance sur la Trinité.

L'alliance spirituelle ne doit pas être éludée. Sur les fonts baptismaux, c'est Josselin Lemarchand qui est déclaré parrain. Il semble bien que cet homme soit décédé sur le Jean de Grâce avant 1700, mais deux de ses frères vivent également au Haut Bout : Olivier et Eustache, tous deux marins. Françoise Girard a accepté d'être la marraine. Elle est sans doute la fille de Jacques et Françoise Lebreton; Jacques figurait sur la liste des inscrits de 1670. Son fils, le seul qui ait, semble-t-il, survécu de ses cinq garçons, Jacques Girard sieur des Clôtures, est également marin. Ce canonnier que l'on voit naviguer dès 1692, poursuivit son activité jusqu'au moins 1730 avec 23 embarquements pour le Petit Nord, Plaisance et Gaspésie.

La famille étant singulièrement élargie, d'autant que remontant d'une génération, il serait loisible de voir où mènent les alliances avec les Naviaut, les Jagoret (dont plusieurs habitent le Haut Bout) et les Rouault (à trou-

18. Le commissaire aux classes, de Gastine, est en poste de 1693 à 1700 . Il a été mandaté à cette fonction pour apaiser les tensions entre administration et armateurs

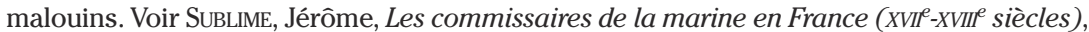
thèse de doctorat d'histoire, sous la direction d'Alain Cabantous, université Paris 1, 2014, vol. 2, p. 30 et vol. 3, p. 104.; JouAn DE KERVENOAËL, Marie-Madeleine, Le système des classes à Saint-Malo au XVII ${ }^{e}$ siècle, thèse de droit dactyl., 1957, Service historique de la Défense de Vincennes, p. 64.

19. Service historique de la Défense de Brest, matricules 1P3 11.

20. Ibid.

21. Ibid. 
ver plutôt à Saint-Coulomb), il reste à avoir une idée de la situation où se trouve la mère de Josselin. Hélène Cliquin est alors âgée de 44 ans. Le couple a eu six enfants parmi lesquels Jacquette, née en 1678, n'a pas survécu. Josselin est né le 15 août 1682 et se trouve à 15 ans être le chef de la famille qui compte Pierre François, né en 1685, et peut-être François (né en 1688), Gilles (1692) et Étiennette (1696) si tant est que ces trois derniers soient encore vivants à ce moment-là. Son mariage aura duré 24 années et elle meurt en 1737 sans s'être remariée. Josselin va lui sur ses 16 ans. Il prétend avoir, ou on lui attribue, une dizaine d'années lorsqu'il embarque en 1698 sur la Faluère ${ }^{22}$. Si le rapport de mer n'a pu être trouvé, le rôle d'équipage apporte suffisamment de renseignements. Cette frégate de 300 tonneaux et 20 canons (la paix ramène la piraterie dans les eaux terre-neuviennes ${ }^{23}$ ) est commandée par Robert Cœuru sieur Desdouetz et appartient au sieur Girard, tous deux étant malouins. Sur les 84 hommes d'équipage, 35 sont originaires du Plessis-Bertrand (41,6\%) : Josselin ne craint rien pour une première fois. On l'a vu, plusieurs proches parents sont à ses côtés : Gilles Cliquin et Robert Grossin ses oncles. S'y trouve également Bertrand Gaultier, son futur beau-frère, de trois ans son aîné. Lui et quelques autres sont les voisins de Josselin : Pierre Gaultier, Guillaume Fumelle et Raoul Bréon vivent au Haut Bout alors que neuf autres vivent dans un périmètre de moins de $2 \mathrm{~km}$, au Valade, à la Gaudichais, à la Baie, la Ville-Gueurie ou la Pintelais.

Le mousse Josselin, qui a appareillé à la fin d'avril 1698, est-il allé à Marseille? Il est seulement indiqué sur sa matricule qu'il a "fait plusieurs campagnes ". Ce sont les rôles d'équipage qui pallient une fois de plus l'insuffisance des indications matriculaires. Il ne réapparaît dans les archives que bien des mois plus tard sur le Saint-Pierre, capitaine Nicolas Brignon, sieur Le Hen. La frégate de 220 tonneaux, 22 canons et 40 hommes, est envoyée de nouveau au Petit Nord. Ayant levé l'ancre le 16 avril 1701, le navire malouin fait sa pêche à l'anse de la Fleur de Lys ${ }^{24}$ jusqu'au 19 octobre puis fait route sur le détroit de Gibraltar, gagnant Marseille le 11 novembre. Seules quelques barriques d'huile de poisson sont déchargées. C'est surtout à Gênes où le Saint-Pierre mouille du 11 décembre au 20 février 1702 puis à Civitavecchia (27 février-15 mars) qu'ont lieu les principaux échanges. Le 19 avril, le Saint-Pierre gagne Saint-Malo avec 2000 quintaux d'alun. Il a ramené tous ses hommes ${ }^{25}$. Il a donc ramené Pierre François Grossin, le jeune frère de Josselin mais celui-ci disparaît des archives, il ne sera jamais classé.

22. Ibid., rôles d'équipage 1P7 70, 1698.

23. HRODĚJ, Philippe, "Les prédations pirates à Terre-Neuve de 1688 à 1725 ", La présence française à Terre-Neuve-et-Labrador : d'hier à demain, Scott JAMIESON, Anne PELTA et Anne Thareau (dir.), Newfoundland and Labrador Studies nº 3, 2015, p. 200-224.

24. Cette petite anse se situe à l'entrée de la baie Blanche.

25. Arch. dép. d'Ille-et-Vilaine, Amirauté 9B 472, rapport de mer. 
Il est logique de penser que Josselin n'a pas passé le Détroit mais a été ramené directement par la saque. En effet, on retrouve le jeune homme sur le César à la même époque. Cette fois les vents le poussent vers le Chapeau Rouge. La frégate jauge 250 tonneaux et porte 20 canons. Duclos Jolif et consort $^{26}$, encore eux, décident d'un départ précoce qui a lieu le 13 mars 1702 de Saint-Malo, quelques semaines avant la déclaration de guerre. Le capitaine, Pierre Léveillé sieur Desvaux, donne les grandes lignes du périple dans son rapport de mer $^{27}$. Le César gagne l'île de Saint-Pierre le 7 mai et mouille à l'anse Bertrand ${ }^{28}$. En quatre mois et demi, quelque 3500 quintaux de morue emplissent la cale. Le 13 septembre, l'Aigle volant le rejoint. Robert Esnoul, son capitaine, qui a été chassé par deux anglais, lui propose de naviguer de conserve pour plus de sécurité. Le 16, les deux morutiers font voile et, le lendemain, à six lieues du cap Miquelon, ils attaquent un navire isolé pensant à une prise française. Il faut rapidement déchanter et, malgré sa promesse, Esnoul, mieux placé, prend la fuite : à bord sont des Cancalais qui abandonnent leurs compagnons ${ }^{29}$. Après deux heures de combat, une cinquantaine d'Anglais montent à l'abordage. Le preneur est la Levrette (capitaine Guillaume Wanthon). À bord, six hommes ont été blessés, Étienne Nicolas de Saint-Cast et Gilles Portier de Cancale (il était sur la Faluère en 1698) ont été tués ${ }^{30}$. Aux dires du capitaine, lui et son équipage sont menés à Newport, au Rhode Island, où ils se perdent de vue. Desvaux Léveillé est embarqué sur un bâtiment portugais qui effectue le " port en port ". Il lui faudra plusieurs mois pour venir témoigner à Saint-Malo, via le Portugal et La Corogne ${ }^{31}$.

À quel moment Josselin a-t-il été échangé? A-t-il été d'abord ramené en Angleterre ou en Irlande? En tout cas, il est indiqué qu'il y a eu un séjour à Boston. Comme sa matricule est incomplète, étant toujours mousse bien qu'ayant à présent 21 ans, il faut consulter celles des autres Cancalais. Étienne Lemonnier ne s'embarque sur le Murinais qu'en décembre 1703,

26. Service historique de la Défense de Brest, rôles d'équipage 1P7 71, 1702. En termes de recherche de capitaines et de bourgeois, la base permettra de mesurer le degré de fidélisation lié à ces personnages-clés.

27. Arch. dép. d'Ille-et-Vilaine, amirauté 9B 474, rapport de mer.

28. Ou anse à Bertrand, à Saint-Pierre, face à l'île aux Marins. C'est précisément à cet endroit qu'ont eu lieu, cette année encore, des fouilles archéologiques sous la direction de Catherine Losier, de l'université de Memorial à St John's.

29. Voici un bel exemple de l'utilisation de cette base. Sur l'Aigle volant, sont embarqués notamment Étienne Lignel et Jean Ricordel, tous deux âgé de 17 ans, le même âge que Josselin. On ne les verra pas être augmentés, car ils sont probablement morts peu après. Se trouvent également Briant Morand, le frère de Robert, sur le César, et Julien Rouillé, canonnier. Surtout, Jean Grossin, oncle de Josselin, est présent! Tous ces Cancalais abandonnent les leurs dans un combat qu'ils savent ne pouvoir leur être favorable. Quel sentiment est le leur? On sait que l'Aigle volant est bien arrivé à Marseille : Julien Rouillé y a débarqué pour aller sur le Grand Joseph, mais, malade, il est mort dans la cité phocéenne.

30. Sans doute Gilles Portier aurait-il pu être parent avec Josselin. Quatre ans plus tard, son fils, Pierre épousera Jeanne Pellé dont la mère est Jeanne Grossin.

31. Arch. dép. d'Ille-et-Vilaine, Amirauté 9B 474, op. cit. 
en compagnie de Josselin Ricordel. Est-ce parce qu'il s'agit d'un voyage à la Chine? Vraisemblablement pas. Briand Morand ne va sur le Canard Doré que le 21 décembre pour faire la course. Thomas Lefevre monte sur le Natal comme maître de chaloupe peu avant le 6 juin 1703 pour une campagne de course. Bertrand Gaultier, lui, se trouve sur le Lyon dès le 2 février pour une traversée à destination de Cadix, Gênes et Livourne. Cet échelonnement n'autorise donc aucune conclusion. En 1704, Josselin est embarqué sur le Saint-Esprit. Il ne s'agit pas du vaisseau du Roi de près de 500 tonneaux portant 74 canons et commandé par Duquesne Guitton au sein de l'escadre blanche du comte de Toulouse, vaisseau qui participe à la bataille de Velez Malaga le 4 août et sur lequel se trouve Jean Lepeigné de Cancale. Ce dernier restera près de trois années à Toulon à armer et désarmer des vaisseaux. Non, Josselin échappe toujours à la vigilance du commissaire aux classes ou bénéficie de sa complaisance. Il se trouve sur le Saint-Esprit, corsaire qui arme à Auray, appartenant au sieur de la Primerais et commandé par le nommé Claude Raoul de Saint-Malo. Ce bâtiment de 300 tonneaux et 36 canons ne se compose que de 42 hommes; il est probable que l'équipage ait été complété par la suite. En tout cas, les gens du Plessis Bertrand sont au nombre de 13 (31 \%), dont Jean Cormier qui figure sur la liste de l'état-major comme maître et l'oncle Robert dont on écrit qu'il a 58 ans, sans doute à cause de ses cheveux gris, alors qu'il n'en a que 45. La campagne est-elle fructueuse, au moins rentable? La base pourra répondre une fois le croisement effectué entre la série $\mathrm{G}^{5}$ du fonds ancien des Archives Nationales, les jugements de prises, et la liquidation desdites prises dont les liasses sont conservées aux Archives départementales des lieux concernés. Normalement, si la course n'est pas lointaine, les commissions sont octroyées pour quatre mois. Josselin est donc disponible dès le mois de mars 1705 . On finira bien par savoir ce que fait ce très vieux mousse jusqu'en 1707; rien ne l'indique sur sa matricule. Sans doute quelque rôle d'équipage finira par parler au fur et à mesure du dépouillement.

Le hasard de recherches à Nantes permet de retrouver Josselin sur le Saint-Esprit en 1707, effectuant un périple entre Cadix et Morlaix via Nantes où est complété le rôle d'équipage ${ }^{32}$. Étrange impression. Il s'agit du même navire, du même propriétaire et du même capitaine. C'est toujours Jean Cormier qui est maître, Jacques Bosquet est passé contremaître. En réalité, pour échapper à l'ennemi, l'équipage malouin de 91 hommes gagne Nantes par voie terrestre en janvier. Aux côtés de Josselin, Robert Cliquin, son oncle $^{33}$. Ce voyage reste mystérieux s'agissant de sa destination, Cadix, où doit se rendre le Saint-Esprit avant de revenir sur Morlaix. Quand on sait que Josselin finit par être rattrapé par le temps et embarque sur le Grand à Brest, dans l'escadre de Du Casse qui quitte Brest le 19 octobre

32. Arch. dép. de Loire-Atlantique, Amirauté, rôles d'équipage C 1191.

33. À noter que les trois frères Cliquin, Gilles (décédé dans les prisons anglaises en 1704), Robert et Olivier, ont épousé trois sœurs Renard, Étiennette, Suzanne et Elizabeth. Suzanne mourra quasi-centenaire juste après la guerre de Sept Ans. 
1707 justement pour l'Amérique espagnole ${ }^{34}$. En juillet, Josselin a perçu une avance pour se rendre dans le grand arsenal du Ponant, 9 livres mensuelles, ce qui est effectivement le traitement d'un matelot en bas de l'échelle. À bord de cette escadre de cinq vaisseaux et trois frégates sont registrés 30 hommes du Plessis-Bertrand sur les 2835 hommes faisant le total des équipages. C'est à bord du Grand (Serquigny) que se trouve la plus grande concentration de ces gens : 10 hommes $^{35}$. Cette fois, il n'y a pas de parents visibles, et c'est bien le cas des embarquements sur les vaisseaux du roi où l'on ne choisit pas! Josselin intègre donc le premier convoi de métal précieux espagnol soutenu par la France puisque l'escadre part au-devant de la flotte de Nouvelle-Espagne qui doit appareiller de La Vera Cruz et la rejoindre à La Havane pour la ramener saine et sauve à Cadix ou dans un port atlantique. Les Français arrivés le 4 janvier 1708 aux abords de la capitale cubaine attendent de longs mois, épuisant leurs ressources; ils sont en revanche épargnés miraculeusement par la maladie, sans doute la fièvre jaune, qui emporte 2000 personnes. L'appareillage général n'a lieu que le 26 juin avec au total 25 ou 26 voiles. Pour un premier embarquement " au service de l'État », Josselin n'est pas déçu. Le Grand et une partie de l'escadre gagnent la rade de Brest le 5 septembre. Chacun s'attache à faire débarquer nuitamment ce qu'il a pu récupérer frauduleusement. Josselin a-t-il eu les moyens de se constituer avant le départ un petit pactole qu'il aurait valorisé? Ce qui est sûr c'est que durant les neuf mois d'absence (à quoi il faut ajouter les déplacements depuis Cancale et l'armement et désarmement qui ne sont pas rémunérés de la même façon), il a perçu 73 livres 2 sols 6 deniers, soit effectivement 9 livres par mois, avec la mention selon laquelle il mérite d'en percevoir 10 ultérieurement. Vingt livres lui avaient été versées en avance, il y a eu par la suite deux paiements par la suite de 30 et 10 livres...; le reste ne sera versé qu'en 1718, dix ans plus $\operatorname{tard}^{36}$.

Son passeport pour s'en retourner au pays a été visé le 11 septembre. Là intervient un vrai problème de chronologie. Le rôle d'équipages du Joyeux est visé le 7 avril 1708 : Josselin se trouve encore à La Havane. En regardant plus attentivement le document, on aperçoit notre marin, âgé de 21 ans et donc toujours très jeune, de taille moyenne avec les chevaux châtains (comme à peu près tout le monde). Le navire est propriété du sieur de la Franquerie et est commandé par Pierre Léveillé, sieur Desvaux, l'infortuné capitaine du César. Sur les 75 hommes d'équipage, 24 sont du Plessis Bertrand (un tiers). On peut signaler que l'état-major est plus représenté qu'à l'accoutumée par des Cancalais : c'est le cas de Philippe Hamon, second, Jacques Buisson, maître, et François Philippe canonnier. Là encore, Josselin navigue en famille : l'oncle Gilles Cliquin, son fils Alexis (qui ne revient probablement pas de ce voyage), l'oncle Robert Cliquin ${ }^{37}$. Le Joyeux

34. Hess, Aurélie et HRODĚJ, Philippe, « Les gens du Plessis-Bertrand sur les vaisseaux du Roi vers les Antilles et le Brésil ", art. à paraître.

35. Le Grand est commandé par Serquigny et porte 500 hommes.

36. Service historique de la Défense de Brest, 1P8 17 à 20.

37. Service historique de la Défense de Brest, 1P7 72, rôles d'équipage 1708. 
part pour le Petit Nord sans que l'on sache pour quelle raison le 16 octobre, il reçoit ce supplément dont fait partie Josselin ${ }^{38}$. Au moins, cela colle-t-il avec les dates écrites dans la matricule puisque ce même mois d'octobre, il est signalé comme étant à Marseille. En réalité, le rapport de mer apporte les morceaux du puzzle manquants. Le Joyeux a bien appareillé de SaintMalo le 17 avril pour atterrir dans la baie de Saint-Antoine, à l'extrême nord de l'île, le 29 mai. Le rapport fait allusion à l'absence d'un " charoy " ou patache que le capitaine n'arrive pas à récupérer auprès de la société de la Sainte-Anne, ce navire se trouvant dans une baie voisine. Mais surtout, le 18 août, des corsaires flessingois sont annoncés qui obligent le capitaine à rallier ses 16 bateaux et se réfugier en fond de baie en abandonnant son matériel. Au bout de 12 jours, le Joyeux gagne nuitamment la haute mer et rentre à Saint-Malo, abandonnant 22000 morues. C'est ce qui avait déjà été embarqué qui sera vendu à Marseille : le retour s'est effectué le 22 septembre, le temps de préparer ce nouveau voyage et permettre à Josselin, venant de Brest, d'embarquer ${ }^{39}$.

On ne sait quand ce navire regagne son port d'attache, probablement en février, mars ou avril 1709. Le 23 juillet, Josselin épouse Olive Gaultier. Il a 27 ans, son épouse 23. Elle étend donc le lignage, entraînant avec elle les Jagoret, les Licornu, les Lignel sans oublier ses parrain et marraine, les Gilbert et les Helbert. Josselin aura le temps de concevoir une fille, Étiennette Marie, qui naîtra 16 mois après les noces, qui ne semble pas avoir survécu et qu'il ne connaîtra jamais. Peu de temps est laissé aux réjouissances. Dès septembre 1709, Josselin est rappelé à Brest avec une solde de 12 livres. Il est libéré assez vite, en décembre, de quoi embarquer sur le Maurepas en avril 1710. Ce navire appartient à Louis Delamart Boudart, est commandé par le sieur de la Guerlerie Alliette. Il jauge 220 tonneaux, porte 22 canons et 91 hommes. Parmi ceux-ci, 18 sont natifs du Plessis-Bertrand (20 \%). Toujours là, l'oncle Gilles Cliquin. Lui reviendra, pas Josselin ${ }^{40}$. Il n'a pas été possible de trouver le rapport de mer. Une apostille très simple indique simplement qu'il n'a pas paru en mars 1711 . On le déclare mort en 1714 et l'on fait son service. Le 3 novembre 1722, à Cancale, Olive Gaultier se remarie avec Noël Duval, de quelques années plus jeune qu'elle. Elle a trois enfants. Seule Thomasse Gillette survit. Olive Gaultier, après 36 ans d'un second veuvage, disparaît en 1769.

Josselin Grossin eut une existence ordinaire de marin qui peut paraître, aux sédentaires forcenés que nous sommes, fabuleuse. Avec la base H3D2, la possibilité d'appréhender ce destin et plusieurs centaines d'autres en quelques clics, sous différentes formes, devient possible à condition de dépouiller plusieurs centaines de milliers de documents et de savoir convenablement les interroger. L'intérêt d'une reconstitution concernant

38. Arch. dép. d'Ille-et-Vilaine, Amirauté 9B 478, rapport de mer.

39. Arch. dép. d'Ille-et-Vilaine, Amirauté 9B 478, rapport de mer.

40. Service historique de la Défense de Brest, 1P7 74, rôles d'équipage 1710. 
"l'embarquer ensemble " est de matérialiser, comme cela vient d'être fait, les bouts de lignages et de voisinages qui, durant de longs mois au sein d'un univers carcéral, expliquent, en plus des amitiés par affinité ou par âge, la patience et la résignation du troisième type humain : le marin. Il est vrai que le temps ne s'écoule pas de la même façon en mer et l'on peut parfois le soupçonner de s'arrêter. Le jeu en vaut la chandelle, il faut l'espérer. Chiotte à chiotte, la pêche sera bonne ${ }^{41}$.

41. Petit à petit (DAGNET, Amand et MATHURIN, Joseph, Le langage cancalais, Saint-Servan, J. Haize, 1904, p. 50). 


\section{RÉSUMÉ}

La réalisation d'une base de données sur les marins du Plessis-Bertrand (c'est-à-dire de Cancale, Saint-Méloir, Saint-Ideuc, Paramé et Saint-Coulomb) pendant le règne de Louis XIV, et jusqu'à la fin du XVIII ${ }^{\mathrm{e}}$ siècle, permet de mettre en évidence l'itinéraire professionnel, l'état sanitaire et les comportements familiaux et démographiques de ces marins. Cette base est constituée à partir de trois sources : les registres de baptêmes, mariages, sépultures des paroisses du Plessis-Bertrand, les registres de matricules et les rôles d'équipage conservés aux Services Historiques de la Défense ou dans les fonds d'amirauté des Archives Nationales.

Grâce au croisement des sources, la base permet l'étude de l'histoire démographique de cette population (l'âge au mariage, le taux de nuptialité, la mortalité, la place de l'activité maritime dans les familles...). La famille Grossin, qui paya un lourd tribut pendant la guerre de la ligue d'Augsbourg, en offre un exemple. L'itinéraire de Josselin Grossin, né le 15 août 1682, montre comment la base permet de reconstituer sa carrière de marin et les relations avec son environnement familial et social : devenu chef de famille à 15 ans, il embarqua dès 1698 comme mousse sur la frégate la Faluère, avec ses oncles Gilles Cliquin et Robert Grossin, son futur beau-frère et des voisins. Des traces de ses embarquements sont trouvées jusqu'en 1710, il est déclaré mort en 1714.

\section{ABSTRACT}

The creation of a database of sailors from Plessis-Bertrand (i.e. Cancale, SaintMéloir, Saint-Ideuc, Paramé and Saint-Coulomb) during the reign of Louis XIV, and until the end of the eigtheenth century, makes it possible to highlight the professional career path, health status and family and demographic behaviour of these sailors. This database is based on three sources: the registers of baptisms, marriages, and burials in the Plessis-Bertrand parishes, personnel number registers and crew rolls kept at the Historical Defence Services or in the Admiralty archives of the French National Archives.

By cross-referencing sources, the database allows the study of the demographic history of this population (age at marriage, marriage rate, mortality, the place of maritime activity in families...). The Grossin family, who paid a heavy price during the Augsburg League War, is a good example. The itinerary of Josselin Grossin, born on 15 August 1682, shows how the database makes it possible to reconstruct his seafaring career and relations with his family and social environment. At the age of 15, he rose to the head of his family and embarked in 1698 on the frigate La Faluère, with his uncles Gilles Cliquin and Robert Grossin, his future brother-in-law and neighbours. Evidence of his various boardings is to be found until 1710. He was declared dead in 1714. 